\title{
Test And analyses of TEM B-field parameter
}

\author{
Yugang Zhao, Zhanguo Lu \\ Beijing Orange lamp Geophysical Exploration \\ Company Limited \\ Beijing, China
}

\author{
Guoqiang Xue, Huasen Zhong \\ Key Laboratory of Mineral Resources \\ Institute of Geology and Geophysics, Chinese Academy of \\ Sciences \\ Beijing, China \\ ppxueguoqiang@163.com
}

\begin{abstract}
In normal TEM sounding, the parameter of secondary induced voltage $V$ signal is collected by using magnetic induced coil, however, the late signal is usually too weak to distinguish deep buried target. In this paper, direct measuring of B-field parameters is used to test its practical effect. Firstly, from the response of $B$-field and $\mathrm{dB} / \mathrm{dt}$ and numerical calculation of different models, we can see that the B-field has superiority in late time detection. Then SM24 TEM system which is used to measure B-field and GDP-32 system which is used to measure induced voltage are compared. With the same observation parameters, $B$-field data is more superiority than induced voltage data. The characteristic frequency of fluxgate sensor is relatively low and it decays slow. In addition, the amplitude of B-field data is larger than $\mathrm{dB} / \mathrm{dt}$ data. It is favorable to measure in late time because of its low effect with low resistivity covered layer. The results show that it is an important direction to solve fine TEM exploration by developing and using fluxgate sensor.
\end{abstract}

Keywords-component; formatting; style; styling; insert (key words)

\section{INTRODUCTION}

We mostly use hollow coil and cored coil form of instrument to acquire electromagnetic field information in order to measure the normalized parameters $\mathrm{V}(\mathrm{t}) / \mathrm{I}$ of secondary induced voltage which is proportional to the rate of change of magnetic field with time $\mathrm{dB} / \mathrm{dt}$, and according it to calculate differential value of induced secondary magnetic field change with time. However, (1) Since $\mathrm{dB} / \mathrm{dt}$ field data decays rapid (its amplitude decays with time), there is a certain degree of distortion in the first and end branch of curve, which is not conducive to high-resolution deep exploration; B-field data decays relatively slow (its amplitude decays with time), and Bfield data is in favor of a good conductor detection. (2) The performance of inductive magnetic probe is usually improved in sensitivity and received signal amplitude by using highperformance core material and increasing the turns of coil, but less contribution to the improvement of SNR. (3)The response of inductive magnetic probe to magnetic field has no relation to the signal frequency, but shows a "frequency-dependent" property, and with the decrease of the signal frequency, the sensitivity of probe greatly decreases.

It is an important way to improve the detection accuracy by obtaining and using the transient electromagnetic B-field parameters. In 1984, Levy introduced a method of obtaining Bfield data by integrating $\mathrm{dB} / \mathrm{dt}$ data. Then McCracken et al. used indirect way, namely, to converted the observed induced voltage into a $\mathrm{dB} / \mathrm{dt}$ field data, and carried out its integration to obtain B-field data after conversion, and used B-field data as parameters to achieve interpretation assignment. In the late 20th century, Smith et al. applied this technology in the aviation transient electromagnetic measurements and interpretation.

With the research and development of detection system with high-temperature superconducting magnetic sensor (SQUIDS), for ground transient electromagnetic measurements, the sensor for direct measurement of B- field component has been further developed. It has carried out a series of field measurements in Australia, Germany, Canada, Japan, China and other regions. James et al., used high-temperature superconducting magnetic sensor in the aviation TEM measurements and achieved a good effect. Chinese scholars Wang Chijun and Chen Xiaodong also carried out the corresponding research and development work, and achieved good results]. However, in the late period, the measured response data by SQUID might turn negative value, therefore, it did not get a good application in the market.

Fluxgate device produced by Australian matching its produced SM24 TEM instrument together is a three-axis fluxgate probes which is mounted on a fixed and non-magnetic platform. It has characteristics for directly observing magnetic flux density B, fast response, a better response on low resistivity body, compact and lightweight. It can carry out three-component observation.Measurement range of magnetic field is from $\mathrm{DC} \sim 4000 \mathrm{~Hz}$, the paper based on the theoretical analysis and comparison with actual measurement results shows that: in the same situation of TEM observation apparatus, B-field data measured by fluxgate sensor has a certain advantage than secondary induced voltage data measured by induction coil, the application and development of B-field magnetic sensor is a good development direction.

\section{B-field Response and Simulation Calculation Analysis}

The traditional TEM access to information is to use hollow coil and the magnetic coil form,which is used for measuring the secondary induced voltage parameters that is proportional to rate of change with time of magnetic field $\mathrm{dB} / \mathrm{dt}$, the induced electromotive force generated by large loop source at the surface of uniform earth is

$$
V(t)=\frac{3 I_{0} \rho_{1} q}{a^{3}}\left[\phi(u)-\sqrt{\frac{2}{\pi}} u\left(1+\frac{u^{2}}{3}\right) e^{-u^{2} / 2}\right]=\frac{d B}{d t} q
$$

In the formula, $u=2 \pi a / \tau ; \phi(u)$ is probability integral. $a$ is radius of emission source loop ; $I_{0}$ is emission current; 
$q$ is effective area of receiving coil. And $\phi(u)=\sqrt{\frac{2}{\pi}} \int_{0}^{u(t)} e^{-t^{2} / 2} d t$.

The relationship of magnetic field parameters and time is as follow:

$$
B_{z}(t)=\frac{u_{0} I_{0}}{2 a}\left[1-\left(1-\frac{3}{u^{2}}\right) \phi(u)-\sqrt{\frac{2}{\pi}}\left(\frac{3}{u}\right) e^{\left(-u^{2} / 2\right)}\right]
$$

Where $u_{0}$ is permeability of free space.

According to Eq. (1) and (2), we can calculate B-field parameter response $\mathrm{dB} / \mathrm{dt}$ on the ground generated by large loop source in condition of uniform half-space $(\rho=100 \Omega \cdot m)$ as shown in Fig. 1 .

As can be seen from Fig.1, the direct measured magnetic field $\mathrm{B}$ parameters has a certain advantages over conventional methods for measuring parameters of rate of change of the magnetic field $(\mathrm{dB} / \mathrm{dt})$ : (1) B- field data decays slower than $\mathrm{dB} / \mathrm{dt}$ field data, B-field data is in favor of good conductor detection. (2) B-field data has a smaller change range than $\mathrm{dB} / \mathrm{dt}$ field data,so its required dynamic range is narrow ,and it is easy to implement in hardware design. (3) the feature point of B-field data appears about 3 times earlier than the feature point of $\mathrm{dB} / \mathrm{dt}$. In conductive feature response, $B$ is stronger than $\mathrm{dB} / \mathrm{dt}$, which can be found earlier in the attenuation curve, which means that the probability of detection will also increase correspondingly.

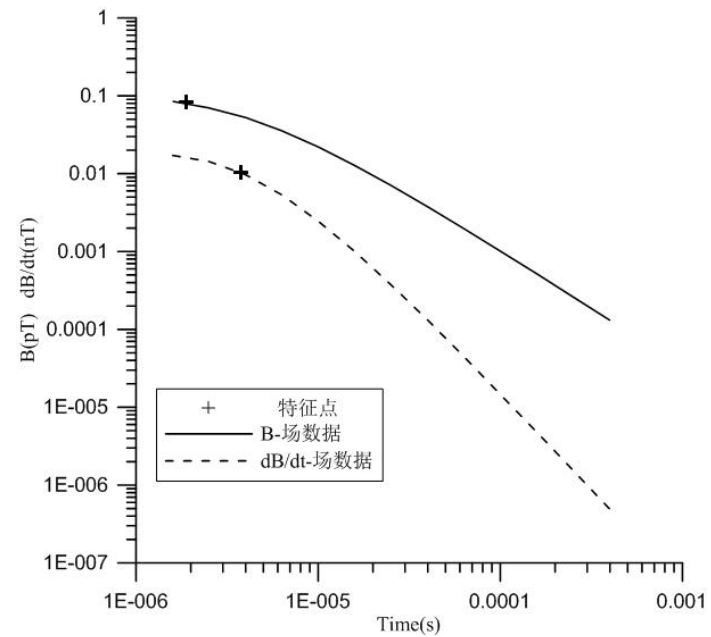

Fig.1 the comparative analysis of B-field data response and $\mathrm{dB} / \mathrm{dt}$ field data response

\section{Measured Data AnAlysis}

\section{A. Equipment And Instruments}

The applied transmitter is for SMARTx4 type transmitter that is high-quality electromagnetic launch system of new generation EMIT with characteristics of reliable operation, high efficiency and safety. it is a set of complete electromagnetic launch system that includes built-in GPS synchronization, which can match any generator, the output power is up to $3.6 \mathrm{KW}$, the maximum continuous operating current is $40 \mathrm{~A}$, the maximum continuous operating voltage is $180 \mathrm{~V}$.

The receiving system SMARTem24 (Figure 2) can effectively provide the highest quality data, and has characteristics of rugged, portable and PC-based electromagnetic system with 16 (8/4 optional) channels, it has become the benchmarking of next generation geophysical electrical measurement system. It has advantages for supporting GPS, quartz clock synchronization, small size, long battery life and other advantages.

The probe of receiving B-field parameters is for SMART fluxgate probe (Fig. 3), which can directly observe three components of magnetic flux density $\mathrm{B}$, frequency range of measured magnetic field is for $\mathrm{DC} \sim 4 \mathrm{kHz}$. The input voltage is from $\pm 12 \mathrm{~V}$ to $\pm 17 \mathrm{~V}$, the output impedance is . Linearity error is $<0.0015 \%$, the most flat frequency response is during 0 $\sim 1 \mathrm{kHZ}$; the biggest error for more than $1 \mathrm{kHZ}$ is up to $\pm 5 \%$, calibration error is $\pm 0.5 \%$, bandwidth is $0 \sim 3 \mathrm{kHZ}$, alignment error (between the reference plane and the $Z$ axis) is $<0.1^{\circ}$.the measurement direction is along direction $Z$, the weight is $160 \mathrm{~g}$, operating temperature is between $-40{ }^{\circ} \mathrm{C}$ and $+70{ }^{\circ} \mathrm{C}$, the measurement range is for plus / minus $70 \mu \mathrm{T}$.

In contrast, the applied instrument for receiving induced electromotive force is GDP-32II, its probe is GDP-32II matching probes. It has almost all of function of electrical measurement for middle and low frequency section, which can be widely used in solid mineral exploration, engineering geophysical exploration and oil and gas exploration, etc. The effective area for measuring electromotive force is 10,000 square meters.

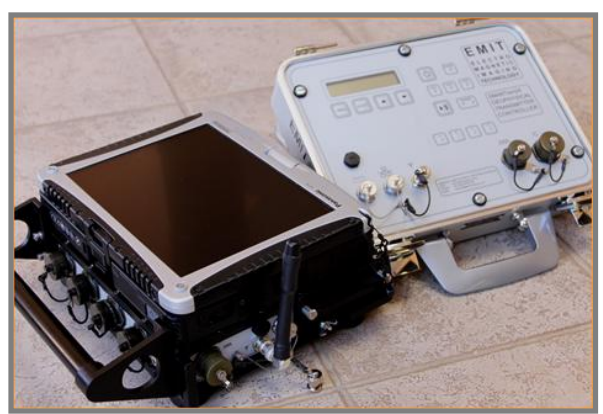

Fig,2 SMARTem24 receiver and frequency controller

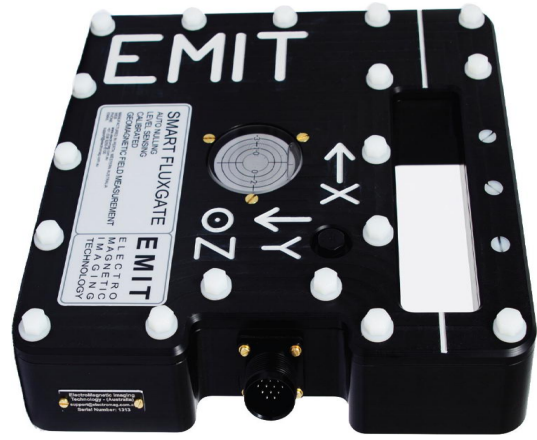

Fig.3 SMART fluxgate probe 


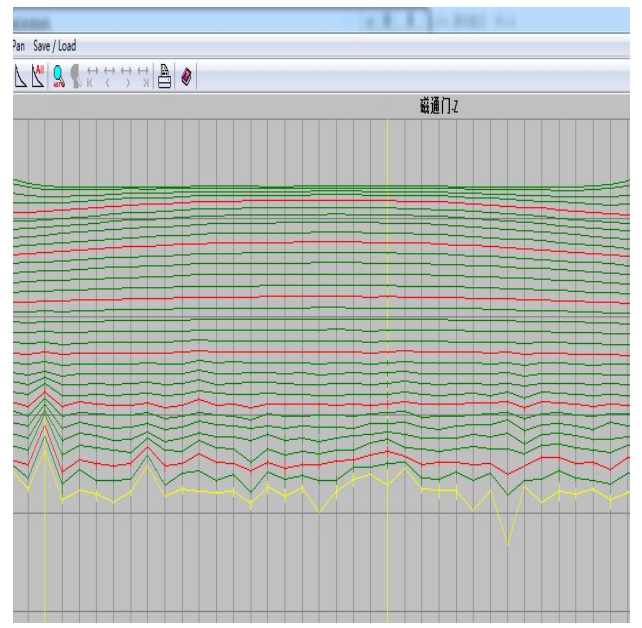

(a) multi-measuring-channel section of B-field parameter

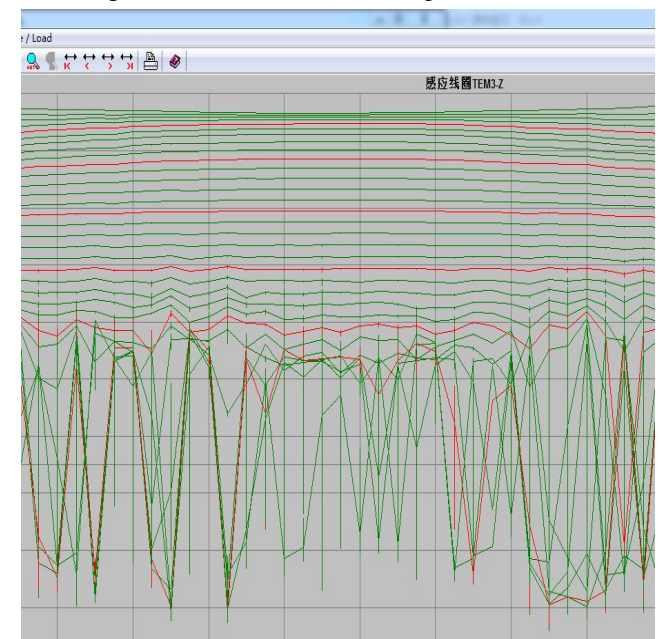

(b) multi-measuring-channel section of induced electromotive force Fig. 5 SM24 and GDP32 result

\section{B. Measured Curve Analysis}

We used SM24 and GDP32 instrument (loop configuration) to detect strong interference areas in the region, used SM24 fluxgate to measure B-field data and GDP32 probe to measure $\mathrm{dB} / \mathrm{dt}$ date,respectively, and the results are shown in Fig.5. From Fig.5 (a), it can be seen that the B- field data obtained by fluxgate is relatively smooth over the entire time series,but the induced electromotive force proportional to the $\mathrm{dB} / \mathrm{dt}$ field data (Fig.(b)) measured by magnetic probe shows a jagged form in the late period,which indicates that, in the strong noise interfering areas, the late $\mathrm{dB} / \mathrm{dt}$ field data is more easily overwhelmed by noise,which is not conducive to probe deep target body.however,the B-field data still has a higher SNR, which is more conducive to probe deep target body.

\section{CONCLUSIONS}

Fluxgate sensor directly measures B-field parameters, but the traditional transient electromagnetic induction coil measures secondary induced voltage to obtain the rate of change of the magnetic field $(\mathrm{d} / \mathrm{dt}$ ) parameters, B-field data obtained by fluxgate decays slow, which will help detection of good conductors.

Compared with the $\mathrm{dB} / \mathrm{dt}$ field data, characteristic frequency of fluxgate sensor is relatively low (often less than 1 $\mathrm{kHz}$ ), decays slow, and B-field data has a greater amplitude range than $\mathrm{dB} / \mathrm{dt}$ field date and a smaller degree of influence by low resistivity overlying strata,which is helpful to the late measurement.

In areas with serious noise, the late $\mathrm{dB} / \mathrm{dt}$ field data is more easily overwhelmed by noise, which is not conducive to probe deep target body. However, the late B-field field data still keeps higher SNR, which is more conducive to probe deep target body.

\section{REFERENCES}

[1] McCracken, K. G., Oristaglio, M. L., and Hohmann, G. W., 1986, A comparison of electromagnetic exploration systems: Geophysics, 51, 810-818.

[2] Eaton, P. A., and Hohmann, G. W., 1987, An evaluation of electromagnetic methods in the presence of geologic noise: Geophysics, 52, 1106-1126.

[3] Smith, R. S., and Annan, A. P., 1998, The use of B-field measurements in an airborne time-domainsystem Part I:Acomparison of B-field with $\mathrm{dB}=\mathrm{dt}$ measurements: Expl. Geophys., 29, 24-29.

[4] Wolfgram, P., and Thomson, S., 1998, The use of B-field measurements in an airborne time-domain system-Part II: Examples in conductive regimes: Expl. Geophys., 29, 225-229.

[5] Duckworth, K., and O' Neill, D., 1989, Detection of a brine conductor under an oil field by means of a fixed transmitter electromagnetic survey using a squid magnetometer: Can. J. Expl. Geophys. 25, 61-73. 